\title{
Effects of Nonlinear Ultrasound Propagation on Output Display Indices
}

Guest Editor

Paul L. Carson, PhD

University of Michigan, Ann Arbor, Michigan

Reviewers for this section were:

Marvin Ziskin, MD, MS

Temple University Medical School, Philadelphia, Pennsylvania

Francis Duck, PhD

Royal United Hospital, Bath, United Kingdom

John Abbott, PhD

Advanced Technology Laboratories, Bothell,

Washington

Ernest Madsen, PhD

University of Wisconsin, Madison, Wisconsin

Adam Shaw, PhD

National Physical Laboratories, Teddington, Middlesex, United Kingdom

\section{Barrie Egerton, MS}

University of Sydney, Lidcombe, New South Wales, Australia

\section{R.T. Hekkenberg, TNO-PG}

Leiden, Netherlands

Diane Dalecki, PhD

University of Rochester, Rochester, New York

Michael Macdonald, PhD

Acuson Corporation, Mountain View, California

[Current address: GE Medical Systems, Milwaukee,

Wisconsin]

Michael Bailey, PhD

Applied Physics Laboratory, Seattle, Washington

Mike Averkiou, PhD

Advanced Technology Laboratories, Bothell,

Washington

Kurt Sandstrom, BS

Advanced Technology Laboratories, Bothell,

Washington

Junru Wu, PhD

University of Vermont, Burlington, Vermont

\section{ABBREVIATIONS}

AIUM, American Institute of Ultrasound in Medicine; MI, Mechanical index; TI,Thermal index; NEMA, National Electrical Manufacturers' Association; ODS, Output Display Standard; TM, Tissue mimicking; KZK, Kuznetsov-Zabolotsakaya-Khokhlov wave equation
$\mathrm{T}$ he six papers in this special section were submitted in conjunction with a workshop sponsored by the AIUM on March 20, 1998, in Boston, Massachusetts. This workshop addressed the effects of nonlinear ultrasound propagation in the measurement medium (water) on the reported output display indices, MI and TI. Key questions of the workshop, addressed in these six papers, included the following: What are the magnitude of the effects? "What approaches might be possible to correct for the effects?" Finally, do the effects appeared significant enough to warrant accounting for them in the measurements and reporting as specified by the 1992 AIUM/NEMA ODS and its later revision and related standards ${ }^{1-3}$ and the Federal Drug Administration 510(k) approval process for diagnostic ultrasound systems. 4

(C) 1999 by the American Institute of Ultrasound in Medicine • J Ultrasound Med 18:27-31, 1999 • 0278-4297/99/\$3.50 
The workshop, consisting of 13 speakers, six other discussants, and 55 registered participants, was conducted by the AIUM Output Standards Subcommittee (cochaired by Gerald R. Harris, PhD, and Chuck Hottinger, PhD) through an organizing committee consisting of Peter D. Edmonds, PhD, SRI International, Menlo Park, California (chairman); Gerald R. Harris, PhD; Christy K. Holland, PhD; Kurt Sandstrom, BS; William D. O'Brien, PhD; Paul L. Carson, PhD; James A. Zagzebski, PhD; and Marvin C. Ziskin, MD, MS. Others who served as discussion leaders or chaired sessions were Mark Schafer, PhD, David Blackstock, PhD, John Abbott, $\mathrm{PhD}$, and Wesley Nyborg, PhD.

Indices defined by the ODS are three TIs and one MI. The TIs (soft tissue, bone-at-focus, and cranial) are estimates of the average temperature rise that will result from exposure of different anatomical sites to ultrasound energy. Also defined is an MI that calculates by very specific rules the maximum peak rarefactional pressure divided by the square root of frequency $\left(p_{r} / \sqrt{ } f\right)$. This index allows some assessment of the likelihood that harm will result from mechanical activity (especially, some form of cavitation). All such estimates are highly dependent on various assumptions, including the assumption that the patient's soft tissues (up to the actual target tissue for TI) have a homogeneous, moderately low attenuation coefficient.

It is important to discuss this topic of nonlinear propagation effects because the achievable peak rarefactional pressure used in the calculation of MI tends to saturate in the measurement fluid (water) at the highest diagnostic outputs. For TI, the frequency content of the pulse and therefore the effective attenuation coefficient of the tissues change as the beam propagates nonlinearly into the tissues. When measurements are made in water and then derated at $0.3 \mathrm{~dB} \mathrm{~cm}^{-1} \mathrm{MHz}^{-1}$ to estimate pressures in an idealized tissue, the acoustic pressures in the idealized tissue are often underestimated. Generation of harmonic frequencies can make the TI an underestimate or an overestimate. In addition, increased particle acceleration and other related phenomena are produced if a shock wavefront is produced. Implications of the latter effects are not well understood.

The paper by Carstensen and associates addresses the overall problems for TI and MI and refers to nonlinear computer modeling of an unfocused transducer. The depths at which $p_{r} / \sqrt{f}$ was maximum varied dramatically as a function of output, thus complicating any analysis. Nonlinear propagation made the estimated error in the ODS calculation (modified for a higher attenuation coefficient of the modeled tissue) underestimate $p_{r}$ by $20 \%$ at even a relatively low source intensity, while linear extrapolation from very low source intensities produced much smaller errors. Christopher, employing the same general computational approach, reported similar results at the meeting. However, more sophisticated calculations allowing for higher peak pressures were employed for his paper in this section and reversed his earlier conclusions. They indicate that, for the particular transducer studied, the ODS underestimates the best estimate of $p_{r} / \sqrt{f}$ by only $8 \%$, whereas linear extrapolation from low source intensities overestimates by $29 \%$. The accuracy and executability which might be achieved with these and other computational methods will probably be critical to precise estimates of $p_{r} / \sqrt{f}$ in vivo.

In Duck's paper, measurements from a commercial imager and linear array show that the method of linear extrapolation from measurements at low outputs produced a calculated $p_{r} / \sqrt{f} 1.6$ times that of the ODS approach. It was assumed that the truth was in between the two and that a major part of the error was in the ODS calculation. Duck proceeds to explain significant work on how to perform the measurements at amplitudes that are low enough for nearly linear propagation but high enough to provide enough signal in the measuring hydrophone and to maximize ease of implementation on current scanners.

The simplest method of imitating ultrasound propagation in tissue is to perform measurements in a TM material. Szabo and colleagues and Macdonald and Madsen report on such measurements in, respectively, tofu and a TM liquid composed of water, evaporated milk, n-propanol, and a preservative. In the symposium, Averkiou suggested that the glycerin and water solutions he has worked with might be more stable than others, and Wu's Ivory Soap, gelatin, and propanol mixture was mentioned. Macdonald and Madsen report here that with a commercial system, the ODS methods underestimated the $p_{r}$ actually measured in the TM liquid by as much as $80 \%$ for long focal depths. Key points required were whether these or other TM liquid media can be standardized adequately and employed effectively for widespread use in the required output measurements. Szabo and colleagues consider a scheme of adjusting transducer voltage drive levels in water to match the $p_{r}$ to that in the attenuating material. They compare it with a consideration of components in the frequency spectrum.

The paper in this section by Egerton and coworkers (presented in the workshop by Barnett) and a separate workshop presentation by Thomenius 
extended the work of Christopher and Carstensen on temperature rise and the TI in unfocused fields and homogeneous tissue. Both employed the computational model developed by Christopher. Egerton and coworkers applied the model to focused fields and reported results of computed field intensities and related temperature rises measured in artificial, homogeneous tissue samples; source intensities were chosen to cover the entire range from closely linear through extremely nonlinear propagation. In his workshop presentation Thomenius estimated temperature distributions in homogeneous tissue and compared results for assumed linear propagation with those for nonlinear propagation. In an extension to plane layered media, he reported on the effect of a fat layer of known thickness on the pressure field and the consequent changes to the temperature distribution for circularly symmetric transducers.

Other presentations at the AIUM workshop addressed simpler other forms of nonlinear propagation calculations than those utilized by Christopher. Averkiou reported on calculations with the approximate KZK equation, which he extended to rectangular apertures. Good agreement was obtained with experiment. While the computational load for determining a broadband field from a rectangular aperture is still prohibitive for real-time calculation, a few hours per beam, that may not be the case in several years. Rielly presented his collaborative work with Baker, Duck, and Humphrey. They utilized the Bergen code, an implementation of the KZK wave equation, adapted for arbitrary frequency dependence of attenuation and for transmission at plane, normal tissue boundaries. Comparisons were made with experimental pressure field measurements in various TM media for a weakly focused $2.25 \mathrm{MHz}$ transducer driven at typical diagnostic amplitudes.

Sandstrom presented verbally and in extensive handouts a wealth of information on characterizing and predicting currently utilized acoustic fields in water. He showed an analysis with a factor of 2 overestimate by linear extrapolation from low amplitudes and a factor of 2 underestimate of pressure amplitude by the current ODS method. Makin, with simulations based on the KZK equation, showed the effects of curved interfaces on transmission and reflection of high intensity ultrasound beams.

Finally, Shaw and Pay reported on temperature rises produced by nonlinear propagation in TM, thermal test objects constructed at the National Physical Laboratories, United Kingdom, to mimic "reasonable worst-case" conditions and used with 16 commercially available pulsed Doppler systems. Predictions and measurements agreed to within $20 \%$.
Shaw proposed an "effective thermal frequency" as a local indicator of nonlinearity. Differences were expected, of course, and found, in fact, in comparisons of these measurements with predictions based on the "average" conditions that ODS models are intended to represent.

Initial conclusions and recommendations were proposed in advance of the symposium, revised somewhat during the day, and voted on in the evening. Consistent with the presentation by Szabo and Worth, efforts were made to create and evaluate the conclusions and recommendations based on criteria of being implementable, fitting into the regulatory environment, being understandable, and fulfilling a compelling need. Since there was little time to discuss changes in the conclusion and recommendation statements and to achieve a consensus on content and wording, the voting had less significance than it might otherwise have had. Particularly, some of the most negative votes and variability in numbers of votes were due to disagreement on wording and lack of understanding of statements being considered. Nevertheless, some sense of the opinions of the participants is given by the votes. Analysis of the votes was performed to determine net positive votes (i.e., number of votes to "agree" and "agree mostly" minus "disagree mostly" and "disagree"). They were also ranked in order of preference, with a "mostly" counting 50\% as much as the full "agree" or "disagree."

Here, in Tables 1 and 2, respectively, the conclusions and recommendations are grouped into various categories of topics. The ranking and net positive votes, net agreement of each of the statements, and the means of those measures are given for each category. Perhaps most important is the highest ranking achieved by any statement in a given category.

As might be expected, there was more disagreement on specific solutions than on the assertions that there is a problem worthy of action or further investigation and that there is a need in any case for improved transfer of information to the user's attention. While Christopher's current theoretical studies show less error from the current measurement methods than the errors presented at the meeting, the assessment that there can be significant errors was probably correct in light of the experimental evidence in TM liquids. The one specific solution (Recommendation Category 5) that received the most attention as well as the highest rating was extrapolation to maximum outputs from measurements made at lower amplitudes where the response is quasi-linear. It was often expressed that nonlinear calculations, when practical and needed, 
Table 1: Rating of Categories of Conclusions

Categories

Ranking of Individual Conclusions

1. There is a need for increased output information and education

2. There is a problem in the measurements

\begin{tabular}{ll} 
& 1 \\
& 2 \\
& 4 \\
& 3 \\
& 6 \\
Average & 8 \\
\cline { 2 - 2 } & 4.6 \\
& 5
\end{tabular}

3. Measurements in an appropriately nonlinear and attenuating fluid would not require corrections

Table 2: Rating of Categories of Recommendations

Individual Recommendations

Net

Ranking Agreement

\section{General Categories}

1. There is a need for increased output information and education

2. There is a problem in the measurements

3. The AIUM should further codify the effects of nonlinear propagation and proceed toward a solution; however, more work is needed before making a change to the ODS

4. Revising the measurements or calculations should not affect regulatory limits on output power for a given beam

\section{Approach That Should Be Taken in Making Corrections or Explored Most Vigorously}

5. Extrapolation from quasi-linear fields

\begin{tabular}{ccc} 
& 1 & 32 \\
2 & 26 \\
3 & 21 \\
& 7 & 7 \\
Average & 18 & 4 \\
\cline { 2 - 3 } & 12.5 & 5.5
\end{tabular}

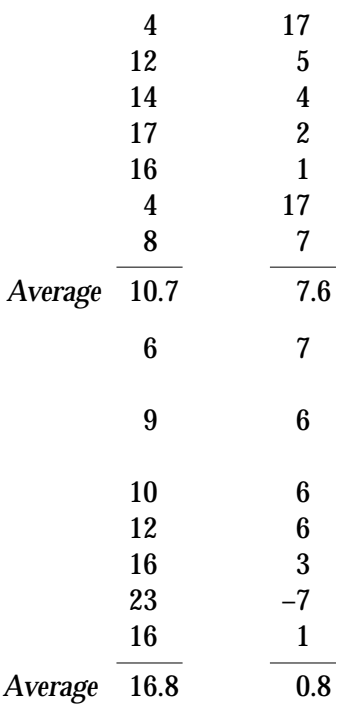

10. Correct measurements based on energy in the harmonics

6. Measurements in a TM fluid

7. Employ nonlinear propagation modeling to correct low or high output measurements and tissue derating

8. Measure with a reduced drive voltage calculated to give tissue-equivalent nonlinear distortion, then boost measurements by ratio of actual to measurement outputs

9. Linear extrapolation from low-amplitude (linear) fields

Average $\frac{13}{16.5} \quad \frac{3}{0.5}$

11. Measure with solid absorbers prior to measurement point

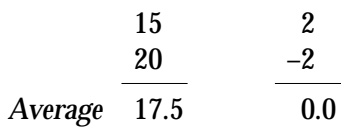


could better be applied to those measurements than to measurements at high amplitude. Measurements made in an appropriately TM medium were thought to be good as a reference for other methods. However, they were thought by many to be impractical for long-term regular use and probably not easily standardized at this time.

It is interesting that no one proposed a recommendation that there is no significant problem and nothing should be done. One relevant proposed recommendation in Category 9 apparently was greatly misunderstood and received the bottom (23rd) rating. The proposal was that no change be made to the ODS but that we undertake measurement and reporting of the reduction (in decibels) of the maximum index value which results from a $20 \mathrm{~dB}$ reduction in output voltage from standard maximum settings. This is equivalent to reporting the levels extrapolated linearly from low amplitude fields, as well as the levels as currently required. This proposal might work well for some time if the displayed outputs were of the linearly extrapolated variety and, if the operator were concerned that the levels were high and might be overreported, he or she could view the currently reported levels.

\section{REFERENCES}

1. AIUM/NEMA: Standard for Real-Time Display of Thermal and Mechanical Acoustic Output Indices on Diagnostic Ultrasound Equipment, Revision 1. Laurel, MD, AIUM Publications, 1998, 64 pp

2. AIUM/NEMA: Acoustic Output Measurement Standard for Diagnostic Ultrasound Equipment. Laurel, MD, AIUM, 1998, 128 pp

3. AIUM: Acoustic Output Labeling Standard for Diagnostic Ultrasound Equipment. Laurel, MD, AIUM Publications, 1998, 32 pp

4. Food and Drug Administration: Information for Manufacturers Seeking Marketing Clearance of Diagnostic Ultrasound Systems and Transducers. Rockville, MD, Food and Drug Administration, Center for Devices and Radiological Health, 1997 